\title{
False Palate Reservoir Denture -A Novel Technique
}

\author{
Dr. Aishwarya Chatterjee ${ }^{1}$, M.D.s, Dr. Debopriya Chatterjee ${ }^{2}$, M.D.s, \\ ${ }^{I}$ Dept of Maxillofacial Surgery, Ramakrishna Mission Seva Prathisthan, Kolkata, West Bengal. \\ ${ }^{2}$ Dept. of Dentistry, IQ City Medical College and Narayana Multispecialty Hospital, Durgapur, West Bengal
}

\begin{abstract}
The subjective feeling of oral dryness is termed xerostomia. Xerostomia is a symptom, not a diagnosis or disease. The term is used to encompass the spectrum of oral complaints voiced by patients with dry mouth. Standard head and neck radiotherapy results in serious, often irreversible damage to the major and minor salivary gland tissues. The resultant profound hypo-salivation leads to serious oral health and functional problems such as intractable root caries, yeast infections and impairment of speech and swallowing. Inadequate quality or quantity of saliva is particularly difficult for complete denture wearers. Extreme discomfort in wearing dentures is a common complaint. Intra-oral devices with reservoirs have been developed that allow the release of saliva substitute into the mouth. Devices for use in edentulous patients where the reservoir has been incorporated within a complete denture have proved successful. Techniques used by previous authors in fabrication of maxillary or mandibular reservoir denture, possibly add to the bulk and cost of the denture. Here a technique is described that is simple, and novel which illustrates the incorporation of a salivary reservoir in a maxillary denture with salivary substitute in it.
\end{abstract}

Key words: xerostomia, reservoir denture, salivary substitutes, complete denture, radiotherapy.

\section{Introduction:}

Xerostomia is defined by the Glossary of Prosthodontic terms as, dryness of the mouth from lack of normal secretions ${ }^{1}$. The subjective feeling of oral dryness is termed xerostomia. Xerostomia is a symptom, not a diagnosis or disease. The term is used to encompass the spectrum of oral complaints voiced by patients with dry mouth $^{2}$.

Xerostomia is a possible side effect associated with more than 400 drugs including antihypertensives, antidepressants, antihistamines, bronchodilators, antiparkinsonians, antispasmodics, anticholinergics and sedatives. Mouthwashes, alcohol, tobacco and caffeine may alter salivary flow or cause dryness of the oral mucosa ${ }^{3}$.

Saliva plays a key role in the maintenance of healthy oral hard and soft tissues and provides essential lubrication during essential oral functions including speech and mastication ${ }^{4}$. The lubricating and chemical buffering effect of saliva is central to the comfort and function of the mouth ${ }^{3}$. Xerostomia is a chronic condition that affects many adults. Autoimmune diseases such as Sjogren's syndrome and rheumatoid arthritis and iatrogenic conditions including drug-related side-effects and radiotherapy for head and neck cancer are common causes of xerostomia ${ }^{4}$.

Standard head and neck radiotherapy results in serious, often irreversible damage to the major and minor salivary gland tissues. The resultant profound hypo-salivation leads to serious oral health and functional problems such as intractable root caries, yeast infections and impairment of speech and swallowing ${ }^{4}$. Inadequate quality or quantity of saliva is particularly difficult for complete denture wearers ${ }^{3}$. Extreme discomfort in wearing dentures is a common complaint ${ }^{5}$.

Whilst systemic and local medications including Pilocarpine can be used to stimulate saliva flow when there is residual gland function, frequently this is not the case and symptomatic relief of xerostomia becomes the primary management strategy. Saliva substitutes such as carboxymethylcellulose, K-Y jelly (Johnson\& Johnson Medical Ltd., Berkshire, UK) and Oral Balance gel (Laclede Inc., Rancho Dominguez, CA) are commonly used. Oral Balance gel has been shown to be an effective palliative treatment that improves patient quality of life. Oral Balance contains enzymes with antimicrobial properties; however, no oral antimicrobial action has been demonstrated in patients, most probably because the dwell time of the gel is relatively short before being swallowed $^{4}$. Intra-oral devices with reservoirs have been developed that allow the release of saliva substitute into the mouth. Devices for use in edentulous patients where the reservoir has been incorporated within a complete denture have proved successful ${ }^{5-12}$. A saliva reservoir is a chamber incorporated into a removable prosthesis that provides a flow of salivary substitute for a certain period of time. Saliva reservoirs range in volume from $2.3 \mathrm{~mL}$ to $5.3 \mathrm{~mL}$ and provide a flow of artificial saliva for 2 to 5 hours ${ }^{6}$. 


\subsection{History and examination:}

\section{Case Report:}

A 40 years old male patient, reported to the Department of Prosthodontics at the College of Dental Sciences, Davangere for replacement of missing teeth (Fig.1). After elicitation of history and a thorough examination, the patient gave a history of radiation therapy for throat cancer 8 months back. Pre radiation therapy, he underwent total extraction of the maxillary and mandibular arch. The patient also complained of difficulty in swallowing and mastication, with frequent drinking of water to ease swallowing. He indicated towards suffering from frequent ulcers. The patient was a first time denture wearer with no history of any prosthesis earlier (fixed or removable). On examination, the mucosa appeared dry (Fig.2). The mouth mirror stuck to the dry buccal mucosa during intra oral examination. The history and examination pointed towards a radiation induced xerostomia. After examination of the history and intraoral findings, a decision was made to construct a reservoir denture containing salivary substitute to aid the patient in his daily activities. The palate was $\mathrm{u}$ shaped and the interarch distance was inadequate to construct a two part reservoir denture. Hence a decision was made to fabricate a false palate reservoir denture for the maxillary arch and a conventional complete denture for the mandibular arch.

\subsection{Denture construction:}

a) Primary impression was made in alginate and an over extended impression was obtained. Custom tray was fabricated and border moulding was done with putty consistency polyvinyl siloxane addition silicone for the maxillary arch (Fig.3) and heavy body consistency polyvinyl siloxane addition silicone for the mandibular arch. Secondary impression was made with monophase polyvinyl siloxane addition silicone material.

b) After the jaw relation and try in stage, wax up of the trial denture was done. In the palatal portion of the maxillary denture, a ledge was created using lingual bar major connector wax, to create a space in the depth of the palatal vault (Fig.4). The wax was sealed in place.

c) Processing was done in the conventional manner and after acrylisation the ledge was obtained in acrylic in the palatal portion of the denture (Fig.5). Thus space was obtained below the ledges in the height of the palate.

d) A latex membrane was sealed to the lips of the ledge (Fig.6) and to the rest of the denture base in the palatal portion. This created the reservoir below the membrane.

e) On the tissue surface of the palatal portion of the maxillary denture, an inlet and outlet was made with $0.8 \mathrm{~mm}$ diameter straight fissure bur, large enough for introducing the artificial saliva and cleaning of the reservoir (Fig.7).

f) The artificial saliva was obtained from a commercial product :

It was introduced into the reservoir using a $5 \mathrm{ml}$ syringe through the inlet and outlet holes already prepared on the tissue surface of the maxillary denture (Fig.8).

g) The patient was instructed about the denture hygiene and refilling and maintenance of the reservoir.

\section{Discussion}

After standard head and neck irradiation for nasopharyngeal carcinoma, salivary hypofunction is an almost ubiquitous lifelong complication ${ }^{4}$. Symptoms in the patient with salivary gland hypofunction are related to decreased fluid in the oral cavity. Patients complain of dryness of all the oral mucosal surfaces, including the throat, and also of difficulty chewing, swallowing, and speaking. The mucosa may be sensitive to spicy or coarse foods ${ }^{2}$.

Saliva substitutes containing thickening agents for longer relief and increased moistening and lubrication of the oral surfaces have been developed. These are available as solutions, sprays or gels and have multiple contents such as carboxymethylcellulose, electrolytes and flavoring agents e.g. wet mouth (ICPA Health Products Ltd), aqwet (Cipla Ltd), saleva (DentAids Ltd.) ${ }^{7}$. However, the main problem is to deliver this substitute constantly into patient's mouth without affecting his normal routine. Where all treatment modalities have proven unsuccessful, the incorporation of artificial salivary reservoir in dentures, has been proposed in such cases. ${ }^{5-12}$

This paper presents treatment of a radiation induced xerostomic patient using a novel technique. The false palate reservoir denture also has potential applications in other cases of xerostomia. The false palate reservoir denture has advantages. It is a simple technique that has no additional steps from those in fabrication of a conventional denture. No detachable parts were involved; hence precision was at a minimum in construction of the reservoir. It allows easy cleaning due to the palatal inlet and outlet holes present in the tissue aspect of the denture. Also it is easy for the patient to refill the reservoir as it is easy for the patient to visualize the level of the salivary substitute through the translucent membrane. The latex membrane that has been used can be easily replaced by the clinician at chair side requiring a minimum of adjustments using cyanoacrylate 
resin. In this type of prosthesis, relining can be done easily with reproduction of the false palate reservoir. Resilient liner may be used to fabricate the saliva reservoir. However, the liner loses its resiliency in 12 to 18 months, and the entire floor of the reservoir may require replacement ${ }^{6}$.

The purpose of the technique is to fabricate a prosthesis that makes swallowing a control mechanism for the flow of saliva substitute. As the patient swallows, the tongue contacts the anterior part of the palate and hence the latex membrane. This creates positive pressure inside the reservoir thus pushing the salivary substitute out of the outlets. When the pressure is relieved, air is sucked in creating a negative pressure. Again the next cycle starts.

The membrane was attached in the palatal vault area. Hence the overall thickness of the palate was increased. The patient had some difficulty in pronunciation of $/ \mathrm{ch} /, / \mathrm{s} /$, and $/ \mathrm{sh} / \mathrm{syllables}$. However they were minimal.

Previous studies ${ }^{6,10}$ suggest that reservoirs in the palatal aspect of maxillary dentures may be useful in treating xerostomia. Techniques used by previous authors ${ }^{6-12}$ in fabrication of maxillary or mandibular reservoir denture, possibly add to the bulk and cost of the denture.

Apart from providing a reservoir denture, the patient was asked to make a conscious effort to consume at least 8 glasses of water, juice or milk daily. Additional recommendations were to include beverages that may produce more saliva such as water with a slice of lemon or lemonade ${ }^{3}$.

\section{Summary}

In summary, fabrication of a novel maxillary complete denture incorporating a false palate saliva reservoir was described. Case selection is important. Before fabrication of a removable prosthesis is started the oral tissues should be ascertained that the denture bearing tissues will accept and support the prosthesis in comfort. The current reservoir denture is a novel design, in that no extra laboratory steps were involved. It has a wide range of usage with a number of products. Further clinical trials are necessary, but the prototype was a success (Fig.9).

\section{References}

[1]. The glossary of prosthodontics terms. J Prosthet Dent 2005; 94:10-92.

[2]. Martin S. Greenberg, Michael Glick. Burket's Oral Medicine Diagnosis \& Treatment. 10th ed. Elsvier; 2003; $236-237$.

[3]. Zarb GA, Bolender CL, Eckert SE, Fenton AH, Jacob RF, Mericske-Stern R. Prosthodontic treatment for edentulous patients. 12th ed. St. Louis: Mosby; 2004. p. 27, 57-58.

[4]. Anne S. McMillan, C.S. Peter Tsang, May C.M. Wong, Andrew Y.L. Kam. Efficacy of a novel lubricating system in the management of radiotherapy-related xerostomia. Oral Oncology (2006); 1 - 7.

[5]. Mendosa AR, Tomlinson MJ. The split denture: a new technique for artificial saliva reservoirs in mandibular dentures. Aust Dent $\mathbf{J}$ 2003;48:190-4.

[6]. Upadhyay Snehal Rashmikant, Kumar Lakshya, and Rao Jitendra. Fabrication of a functional palatal saliva reservoir by using a resilient liner during processing of a complete denture. J Prosthet Dent 2012; 108:332-335.

[7]. Pattanaik Bikash, Pattanaik Seema. Prosthetic Rehabilitation of A Xerostomia Patient With A Mandibular Split Salivary Reservoir Denture. Annals and Essence of Dentistry 2010.

[8]. Vergo TJ Jr, Kadish SP. Dentures as artificial saliva reservoirs in the irradiated edentulous cancer patient with xerostomia: a pilot study. Oral Surg Oral Med Oral Pathol 1981; 51:229-233.

[9]. Sinclair GF, Frost PM, Walter JD. New design for an artificial saliva reservoir for the mandibular complete denture. J Prosthet Dent 1996;75:276-280.

[10]. Toljanic JA, Zucuskie TG. Use of a palatal reservoir in denture patients with xerostomia. J Prosthet Dent 1984;52:540-544.

[11]. R. B. Hallikerimath, Manish Jain. Managing the Edentulous Dry Mouth: The Two Part Mandibular Denture. J Indian Prosthodont Soc (Jan-Mar 2012) 12(1):51-54.

[12]. Nupur Dabas, Sumit Singh Phukela, and Harish Yadav. The Split Denture: Managing Xerostomia in Denture Patients: A Case Report. J Indian Prosthodont Soc. 2011 March; 11(1): 67-70.

\section{Legend for photographs:}

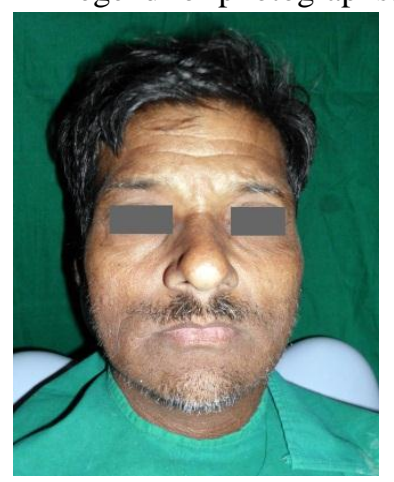

Fig 1.. pre-operative frontal view 


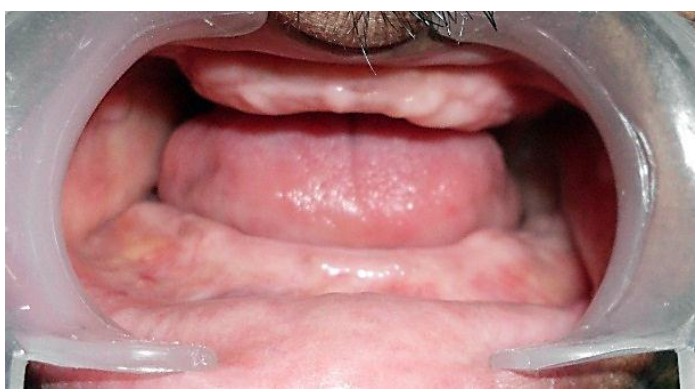

Fig. 2 - intraoral photo showing dry mucosa

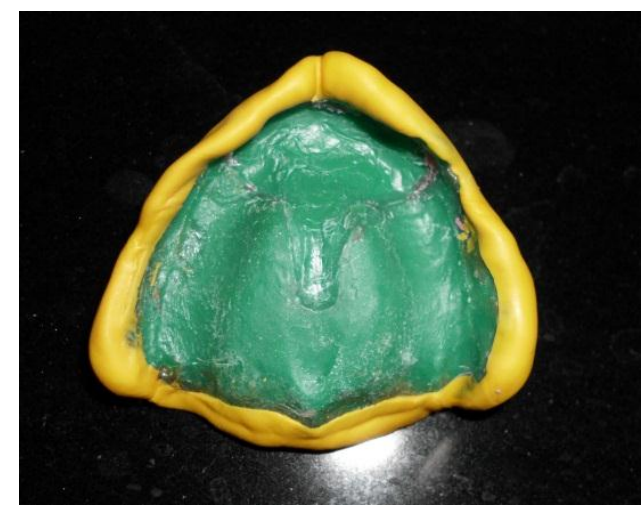

Fig. 3 - putty border moulding

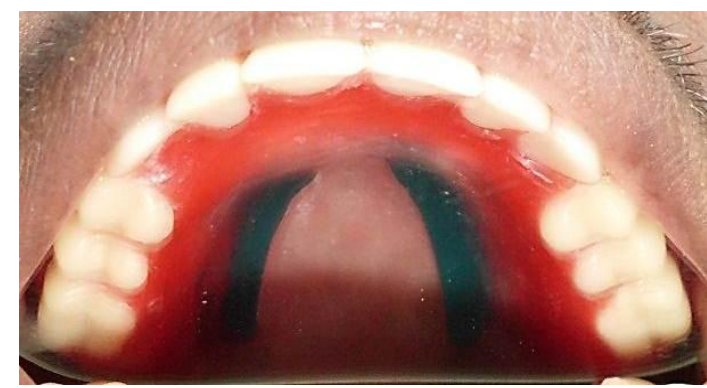

Fig.4 - wax up with lingual major connector ledge created.

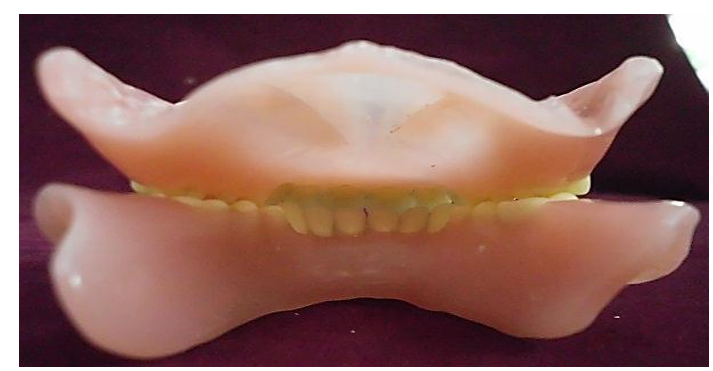

Fig. 5 - acrylised dentures with ledge visible in maxillary denture

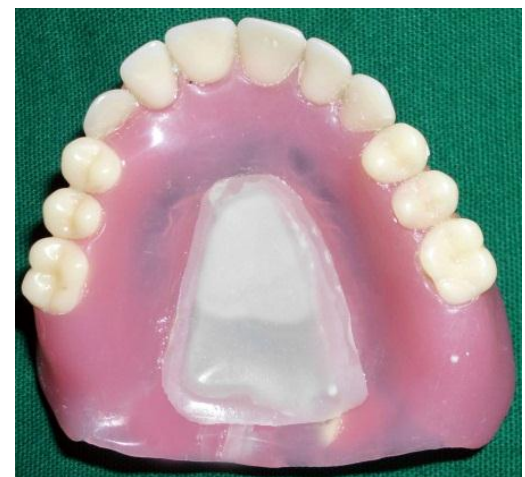

Fig. 6 - latex membrane in place over lips of ledge with artificial saliva in reservoir 


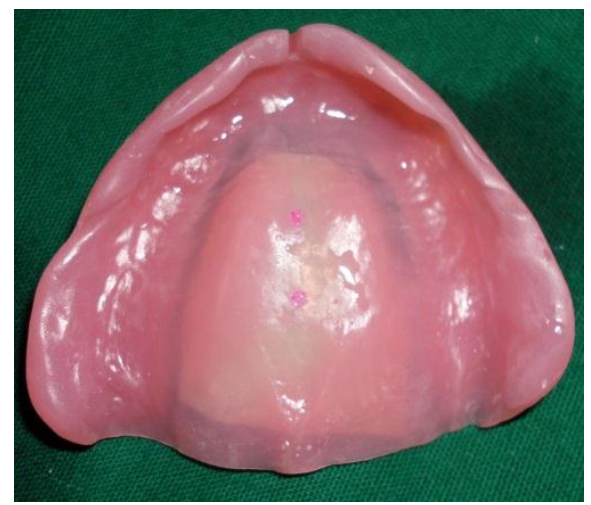

Fig. 7 - inlet and outlet holes in palatal aspect of maxillary denture.

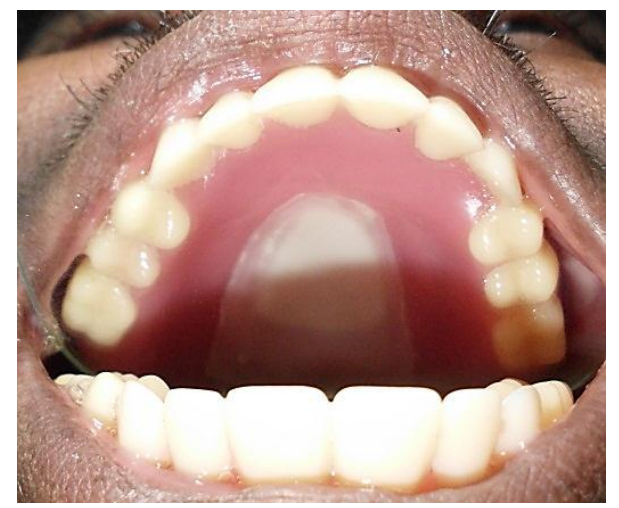

Fig. 8 - latex membrane reservoir in place intraorally.

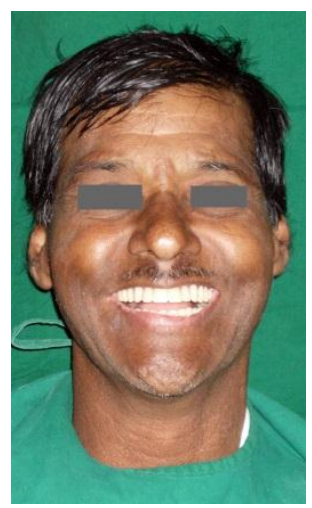

Fig. 9 - postoperative frontal view. 